\title{
CHALLENGES TO THE FERMILAB LINAC AND BOOSTER ACCELERATORS*
}

\author{
R. Webber ${ }^{\dagger}$, FNAL, Batavia, IL 60510, USA
}

\begin{abstract}
A report on the challenges confronting the Fermilab Linac and Booster accelerators is presented. Plans to face those challenges are discussed. Historically, the Linac/Booster system has served only as an injector for the relatively low repetition rate Main Ring synchrotron. With construction of an $8 \mathrm{GeV}$ target station for the $5 \mathrm{~Hz}$ MiniBooNE neutrino beam and requirements for rapid multi-batch injection into the Main Injector for the NUMI/MINOS experiment, the demand for $8 \mathrm{GeV}$ protons will increase more than an order of magnitude above recent high levels. To meet this challenge, enhanced ion source performance, better Booster orbit control, a beam loss collimation/localization system, and improved diagnostics are among the items being pursued. Booster beam loss reduction and control are key to the entire near future Fermilab high energy physics program.
\end{abstract}

\section{INTRODUCTION}

Approved experimental programs under construction at Fermilab call for $8 \mathrm{GeV}$ protons at rates far exceeding past performance of the Linac and Booster, the Fermilab Proton Source. Compared to the original Fermilab Main Ring, the larger acceptance and faster cycle time of the Main Injector allow operation at higher injected pulse intensities and higher average pulse rates for antiproton production and for experiments like MINOS [1], the high energy, long baseline neutrino experiment. Also, an experiment is being constructed that will, for the first time, take $8 \mathrm{GeV}$ protons directly from Booster on target. That experiment, MiniBooNE [2], expects beam pulses at a $5 \mathrm{~Hz}$ rate.

Implications of increased proton delivery requirements include: upgrades of pulsed power systems to permit equipment operation at sustained pulse rates in excess of $8 \mathrm{~Hz}, 40 \%$ increase in peak beam pulse intensity, coping with potentially higher prompt radiation in surface level buildings and grounds around Booster, and more residual activation and contamination of accelerator components.

\section{DEMANDS VS. PERFORMANCE}

Fermilab's schedule calls for Tevatron Collider Run II to run from now through at least 2007. Run II antiproton production initially requires $5 \mathrm{E} 12$ protons per pulse (ppp) each 1.47 seconds. Two pulses of that same intensity will

\footnotetext{
*Work supported by the U.S. Department of Energy under contract No. DE-ACO2-76CH03000.

†webber@fnal.gov
}

be required every 1.6 seconds when slip-stacking is implemented in the Main Injector. MiniBooNE, scheduled to turn on in mid-2002 and run concurrently with Run II for longer than one year, requires $5 \mathrm{E} 12 \mathrm{ppp}$ at an average rate of $5 \mathrm{~Hz}$. In late 2004, MINOS comes on-line expecting 3.5E20 protons per year from Main Injector. Operation of MINOS with Run II antiproton production may require Main Injector taking six beam pulses of up to 6.5E12 from Booster each 2 seconds. A follow-on of MiniBooNE may also run in this era.

Figures 1 and 2 show how these scenarios play out for the Proton Source. Figure 1 displays the hourly proton demand and indicates both the historically highest proton delivery rate (mid-70's Main Ring fixed target era) and that achieved during Tevatron Collider Run I (six years ago). An increase of more than a factor of five above the historical high is requested. It is relevant to note that that high was achieved within radically different radiation constraints.

Figure 2 shows the anticipated per pulse beam intensity and pulse rate needed to meet the hourly proton demand. The proposed 7E12 ppp operating intensity is to be compared to the current Booster record of 5.7E12. Typical Booster operation is presently near 5E12 ppp with Linac regularly running 55 to $58 \mathrm{~mA}$, near it's record $\mathrm{H}^{-}$beam current of $62 \mathrm{~mA}$. With protons, the Linac has been demonstrated capable of accelerating currents in excess of $90 \mathrm{~mA}$. [3] Average beam pulse rates up to $8 \mathrm{~Hz}$ will be required. Linac regularly accelerates beam up to $66 \mathrm{MeV}$ at $15 \mathrm{~Hz}$ for neutron therapy operation, however the historical high for Booster is only around $3 \mathrm{~Hz}$. The dropoff in proton demand in 2006 reflects completion of the MiniBooNE experiment as currently envisaged.

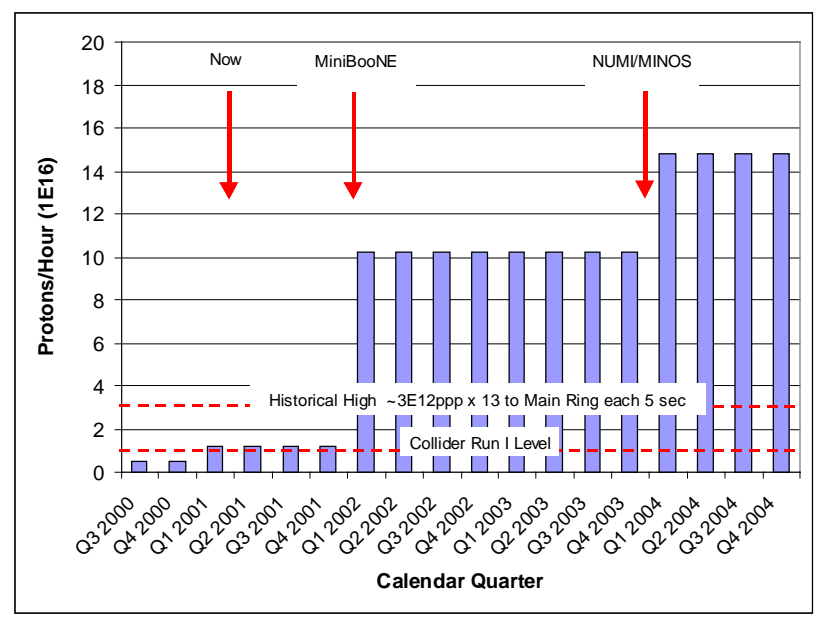

Figure 1. Near Term Proton Demand 


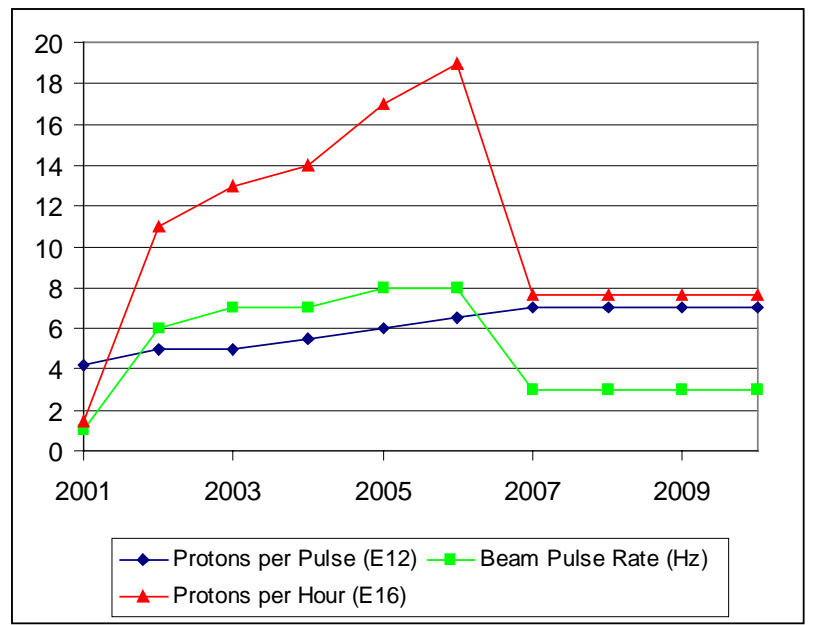

Figure 2. Proposed $8 \mathrm{GeV}$ Proton Production by Year

\section{IMPLICATIONS}

\subsection{Power Equipment Pulse Repetition Rate}

The projected beam pulse repetition rate has no impact on PreAccelerator, ion source, or Linac power equipment; it has always pulsed continuously at $15 \mathrm{~Hz}$. In Booster, only the main magnet circuit operates continuously at that rate. The Booster RF systems and injection and extraction devices are pulsed only as needed for beam and are not necessarily designed for continuous operation. [4]

To achieve the $8 \mathrm{~Hz}$ beam rate, replacement or upgrade is required of the extraction septum magnets and power supplies and the injection orbit bump magnets and power supply. Each of these is a multi-kiloampere pulsed power system with magnets fabricated with "in-vacuum" coils and laminations to meet fast rise/fall time requirements. The 30 year old Booster RF systems are designed for the required repetition rate, but reliability is a concern.

\subsection{Beam Pulse Intensity}

$\mathrm{H}^{-}$injection into Booster permits some flexibility as to how the required injected beam current is obtained. Empirically, Booster operates with lower beam loss and higher acceleration efficiency when the Linac delivers high beam currents, i.e. fewer injected turns of high Linac current are better than more turns of lower current. The current available from the $\mathrm{H}^{-}$ion source [5] presently limits Linac output current.

Figure 3 shows typical Booster beam signals through the $33 \mathrm{msec}$ acceleration cycle for different intensities, the highest approaching 5E12 ppp. Causes of inefficiency and beam loss include: limited aperture, poor orbit control, space charge effects, imperfect RF capture of injected beam, transverse resonances, transition crossing, head-tail instabilities, and longitudinal coupled bunch instabilities.

\subsection{Beam Loss and Radiation Issues}

Radiation due to uncontrolled beam loss in Linac and especially Booster sets the critical path to meeting beam

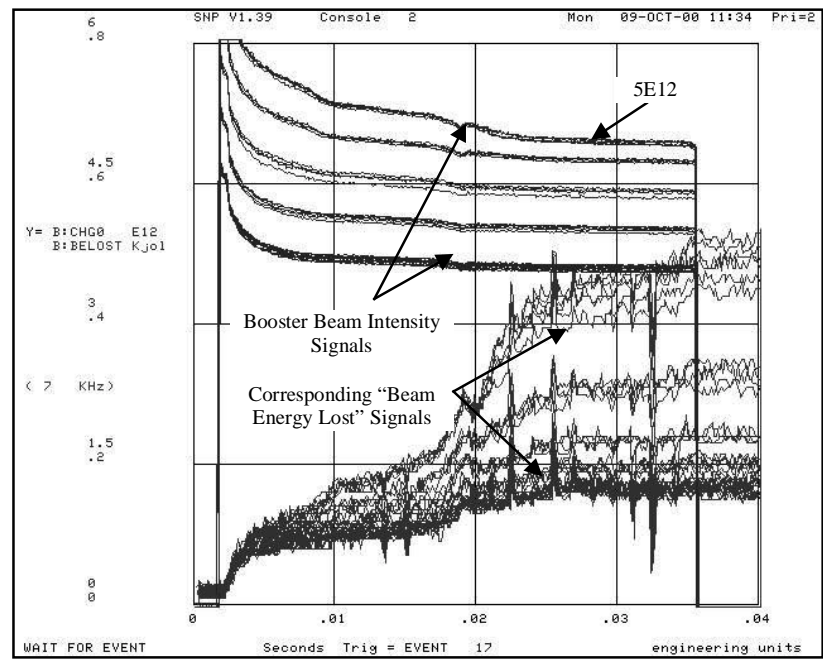

Figure 3. Typical Booster Intensity and Loss Signals

Through the Acceleration Cycle at Different Intensities

demands. There is inadequate shielding around part of Linac and most of Booster to passively meet Fermilab radiation safety standards. Hence, an array of interlocked radiation detectors sets the envelope within which the machines must operate. The potential to create unacceptable levels of residual radiation or contamination of beam line components will be very real at the projected beam rates. It is as yet unclear which aspect of the radiation issue will be the operationally limiting effect.

Safety conditions are much more constrained than in the 1970's for several reasons: construction over the years has greatly increased the number of offices and tech areas in close proximity to the Booster, the original shielding design for the machine has been adversely affected, the extraction point has been relocated to a more sensitive location for transport of beam to the Main Injector, and radiation regulations are now much tighter.

The 'beam energy lost' signal in Figure 3 shows typical Booster losses, all uncontrolled, of up to 500 joules per cycle at 5E12ppp. At $8 \mathrm{~Hz}$, this extrapolates to $4 \mathrm{~kW}$ or $8.5 \mathrm{~W} / \mathrm{meter}$ around the $470 \mathrm{~m}$ circumference, nearly an order of magnitude higher than the Proton Driver [6] design goal of $<1 \mathrm{~W} / \mathrm{m}$ uncontrolled loss for hands-on maintenance. Figure 4, which shows the non-linearity of losses vs. intensity, provides an additional indication of the difficulties to be encountered to achieve 7E12 ppp. Extraction losses are not included in either Figs. 3 or 4.

Figure 5 shows typical signals from the 50 interlocked Booster radiation detectors during a well-tuned operating period of 5E15 protons per hour (pph) at 4E12 ppp last year. The numbered vertical scale is normalized to the hourly rate trip point of each detector and the data is linearly scaled up to $1.2 \mathrm{E} 16 \mathrm{pph}$ (Run II only rate). There appears to not be a problem operating within the hourly limits for Run II. The line at 0.125 on the plot however shows that numerous locations exceed the trip level when scaled to $1 \mathrm{E} 17 \mathrm{pph}$, approximately the rate for Run II plus MiniBooNE. To make matters worse, the detectors 


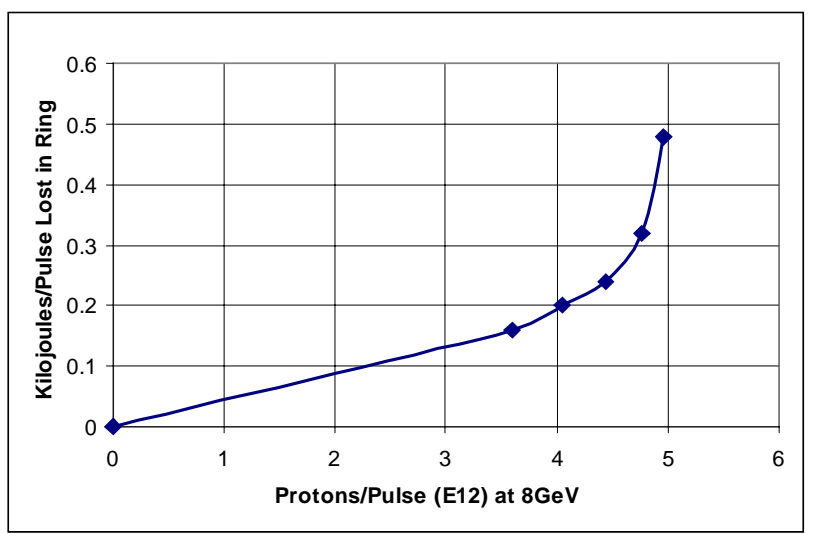

Figure 4. Relation Between Beam Energy Lost in Booster and Final Intensity

indicated by 'dumbbells' are in locations with permissible yearly rates corresponding to just $20 \%$ of the allowed hourly dose rates. Clearly, successful efforts to reduce and/or control the location of beam loss are crucial.

\subsection{Reliability and Availability Issues}

The programmatic yearly goals for protons on target imply nearly continuous 12 month operation at the prescribed intensities and pulse rates. Traditionally high machine availability and accelerator component reliability will remain important, even in the face of the stress imposed by higher continuous pulse rates, higher beam intensities, and significantly higher radiation loads.

Failure of tunnel components will be especially intolerable. High radiation levels and increased likelihood of contamination may force cool-down periods prior to tunnel access. ALARA concerns will require increased Radiation Safety Officer review and coordination of activities, leading to longer downtimes and reduced machine availability. RF systems and existing injection orbit bump magnets present particular vulnerabilities.

\section{CURRENT ACTIVITIES}

- An ion source development program is beginning with the goal of achieving a higher current, brighter Linac beam for injection to Booster. [5] [7]

- Installation of controls to ramp the Booster dipole corrector magnets to facilitate orbit control to higher energies has begun. The correctors now run DC only.

- Booster BLM data acquisition improvements have been made to facilitate beam studies, provide time averaged data, and track operating trends.

- Operation of Booster with a kicker rise time gap in the beam has already greatly reduced extraction radiation problems. Further work to synchronize the gap on multi-batch Main Injector cycles is needed.

- A prototype Booster RF cavity with enlarged aperture is in fabrication. The cavities now present aperture limits at nine straight sections in Booster.

- A new prototype RF power amplifier that will reduce tunnel component failure is in operational testing.

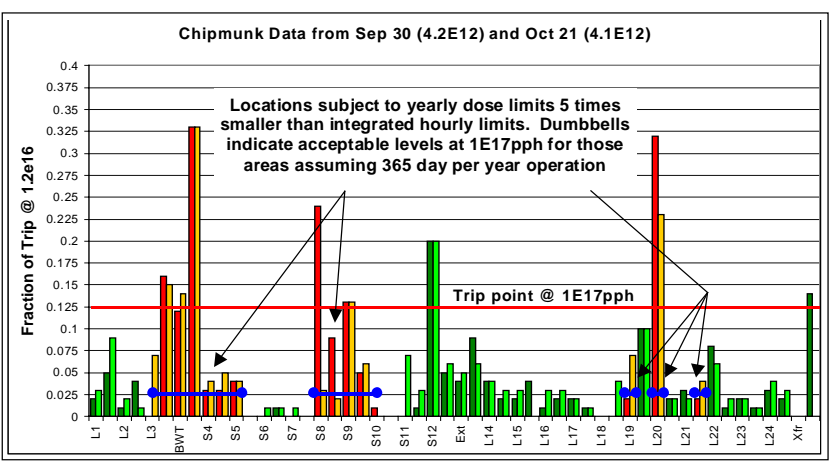

Figure 5. Booster Interlocked Radiation Detectors Signals Normalized to Trip Levels

- Additional shielding will be installed in critical locations around Booster; though options are limited

- A beam collimator system to localize beam loss to a controlled location in Booster amenable to additional shielding is designed and partially installed. [8]

- A new extraction septum magnet, designed for continuous $15 \mathrm{~Hz}$ operation, is fabricated and nearly ready for test. New extraction septum and injection orbit bump power supply designs are in progress.

\section{CONCLUSION}

Demand for $8 \mathrm{GeV}$ protons will increase by an order of magnitude in the coming year. Booster will be asked to deliver more protons per year than it has accelerated in its entire 30 year lifetime. While considerable effort has been spent over the last two years to specify a Booster replacement [9] and produce a comprehensive Proton Driver design report, even the most optimistic scenario does not have a replacement on line in less than six years. For the foreseeable future, the Fermilab program must continue to rely on the existing machines and the difficult challenges facing the Proton Source must be met head-on.

I wish to acknowledge the ongoing efforts of the entire Fermilab Proton Source Department. Thanks to Peter Kasper for supplying Figure 5.

\section{REFERENCES}

[1] NUMI/MINOS, http://www-numi.fnal.gov:8875.

[2] MiniBooNE, http://www-boone.fnal.gov/.

[3] M. Popovic, et al. "High Current Proton Tests of the Fermilab Linac", Linac 2000, Monterey, California.

[4] R. Webber, "Report on Booster High Pulse Repetition Rate Tests ...", Fermilab TM-2074, March, 1999.

[5] V. Dudnikov, "30 Years of High-Intensity Negative Ion Sources for Accelerators", this conference.

[6] W. Chou, editor, "The Proton Driver Design Study", Fermilab TM-2136, December, 2000.

[7] D. Young et al., "Low Energy Improvements to the Fermilab 400-MeV Linear Accelerator", this conference.

[8] A. Drozhdin et al., "Beam Loss Collection in the Fermilab Booster", this conference.

[9] W. Chou et al., "Summary of the Fermilab Proton Driver Design Study," this conference. 